\title{
Rapid Fluctuations in Rat Barrel Cortex Plasticity
}

\author{
Irina A. Erchova and Mathew E. Diamond \\ Cognitive Neuroscience Sector, International School for Advanced Studies, 34014 Trieste, Italy
}

Neuronal populations in the sensory cortex exhibit fluctuations in excitability, and the present experiments tested the hypothesis that these variations coincide with peaks and troughs in cortical modifiability. The activity of multiunit neuronal clusters under light urethane anesthesia was recorded through 100-microelectrode arrays implanted in the infragranular layers of rat barrel cortex. Spontaneous activity was characterized by "bursts" of spikes, synchronized across the barrel cortex. This allowed activity at one selected electrode to be taken as a reliable monitor of widespread cortical bursts. We used spikes at the selected electrode to trigger stimulation of two pairs of whiskers during a 50 min conditioning procedure: (1) for the "burst-conditioned" whisker pair, each stimulus was delivered $1 \mathrm{msec}$ after the triggering spike, activating cortex coincident with the burst; and (2) for the "interburst-conditioned" whisker pair, each stimulus was delivered $300 \mathrm{msec}$ after the triggering spike, activating cortex during the trough between bursts. The cross-correlation between cortical neurons in the pairs of columns matching the stimulated whisker pairs was estimated after the termination of the conditioning procedure. Conditioning produced a twofold increase in the degree of co-firing between infragranular neurons in columns receiving burstconditioned costimulation but no significant change in connectivity between infragranular neurons in columns receiving interburstconditioned costimulation, although the two pairs of columns received an equal number of sensory inputs. These findings suggest that the strength of co-activity between columns in the barrel cortex can be modified by sensory input patterns during discrete, intermittent intervals time-locked to bursts.

Key words: burst; cortical rhythms; whisker; vibrissa; sensory; anesthesia

\section{Introduction}

Cyclical oscillations in neuronal firing, coordinated across widespread regions of the cortex, are a characteristic common to many different brain states: they are present in anesthetized, in sleeping, as well as in alert animals under a variety of behavioral conditions. In general, during sleep and anesthesia states, the oscillation amplitude is more profound and the rhythm is slower $(0.5-3 \mathrm{~Hz})$; the EEG correlates of these states are high-voltage, low-frequency waves (Tobler, 2000). In the somatosensory cortex of rats, the focus here, urethane anesthesia produces activity resembling slow wave sleep during which single-unit bursts are synchronized across distant cortical sites (Fox and ArmstrongJames, 1986). During alert, waking states, the oscillation amplitude is often shallow and the rhythm is faster; here, the EEG is characterized by low-voltage, high-frequency waves (for review, Siegel and Rogawski, 1988). Individual neurons in different brain areas have a different pattern of activity depending on the sleep stage and the phase of the EEG wave cycle (Nunez, 1996; Manns et al., 2000; Steriade et al., 2001).

Many studies have focused on the mechanisms that generate rhythmic, synchronized activity (Steriade et al., 1990, 1993;

Received Aug. 28, 2003; revised May 12, 2004; accepted May 17, 2004.

This work was supported by European Community Grant IST-2000-28127, Telethon Foundation Grant GGP02459, J. S. McDonnell Foundation Grant 20002035, and the Human Frontiers Science Programme.

Correspondence should be addressed to Dr. Mathew E. Diamond, Cognitive Neuroscience Sector, International School for Advanced Studies, Via Beirut 2/4, 34014 Trieste, Italy. E-mail: diamond@sissa.it.

I. A. Erchova's present address: Unité des neurosciences Intégratives et Computationelles, Institut de Neurobiologie Alfred Fessard, Bâtiment 33, 1, Avenu de la Terrasse, 91198 Gif-sur-Yvette, France.

DOI:10.1523/JNEUROSCI.1202-04.2004

Copyright $\odot 2004$ Society for Neuroscience $\quad$ 0270-6474/04/245931-11\$15.00/0
Llinas and Pare, 1991; Timofeev et al., 2000), but uncovering the functional meaning of cortical oscillations has remained elusive. Leading theories include information transfer between cortical regions (Hoffman and McNaughton, 2002) binding within the zones contained within one sensory cortical area, binding among different the cortical areas subserving the same sensory modality (Freiwald et al., 1995; Kreiter and Singer, 1996), and even binding among distant regions of cortex as a substrate for consciousness (Pare and Llinas, 1995; Llinas et al., 1998).

In a recent study (Erchova et al., 2002), we used 100microelectrode arrays to characterize the behavior of neuronal populations in the "barrel cortex" (the cortical whisker representation) of lightly anesthetized rats. The spontaneous firing pattern of neurons consisted of clusters of spikes ("bursts") separated by periods of low spike density, and the burst and interburst intervals were correlated across the entire barrel field. The key observation was that, measured by the magnitude and spatial extent of response, neurons showed the greatest responsiveness to sensory inputs (single-whisker deflections) when the stimulus was delivered during bursts and the least responsiveness when the stimulus was delivered during the interval between bursts. Thus, during bursts, there seemed to be the greatest opportunity for individual neurons to integrate inputs from multiple whiskers, creating conditions favoring timing-dependent forms of Hebbian (Hebb, 1949) synaptic plasticity. Based on these observations, we hypothesize that bursts may modulate plasticity, enabling cortex to be modified by patterns of sensory input preferentially at discrete intervals aligned with the bursts.

Testing the hypothesis requires comparing the cortical modification induced by sensory inputs delivered when cortex is at the 


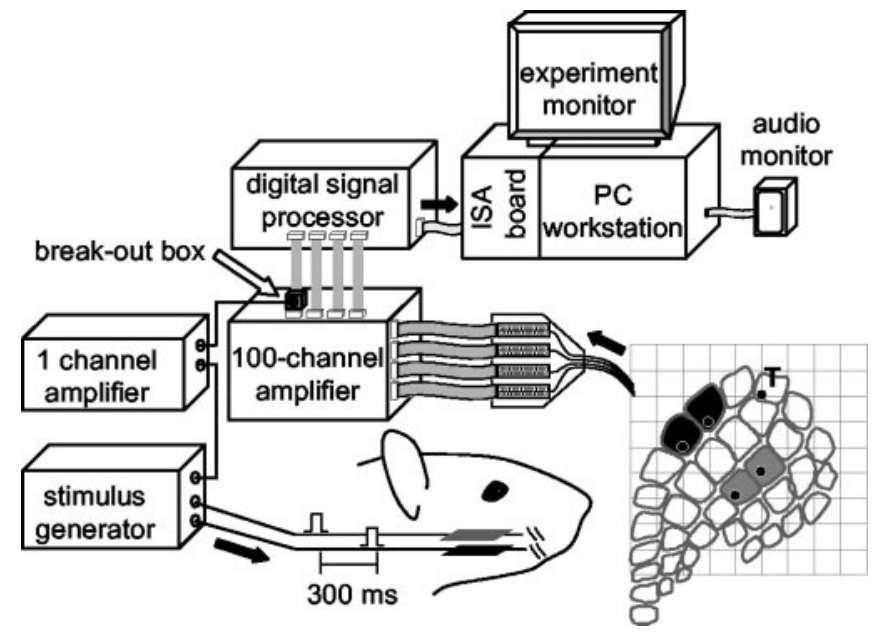

Figure 1. Recording and stimulation conditions. The plot at the bottom right indicates the $10 \times 10$ electrode array position relative to the cortical barrel field for this experiment (R20). Spikes from channel n.76, located in column $\delta$, were led via a "break-out box" (Digikey AK946, Thief River Falls, MN) to a one-channel variable gain amplifier (Horowitz and Hill, 1990), the output of which led to a stimulus generator (A.M.P.I.). This produced pulses that were delivered to piezoelectric wafers situated in contact with two pairs of whiskers. Cortical columns matching the stimulus sites $\left(C_{2}-C_{3}\right.$ and $\left.E_{2}-E_{3}\right)$ are shaded in gray and black, respectively. The site of the trigger electrode is indicated by $\mathrm{T}$.

peak of the oscillation as opposed to when it is at the trough of the oscillation. As an input pattern, we selected paired whisker stimulation (simultaneous deflection of two adjacent whiskers) because this induces receptive field plasticity and changes in the effective connectivity between cortical barrel columns in awake, freely moving rats (Lebedev et al., 2000). To determine whether cortical modifiability is determined by the precise timing of sensory inputs in relation to the instantaneous cortical state, we repeatedly deflected one whisker pair during bursts, while deflecting a second whisker pair during interburst intervals, across a 50 min conditioning procedure (Fig. 1). We predicted that the barrel columns of the first whisker pair would become more strongly linked, having received the conditioning stimuli when plasticity was facilitated, whereas the barrel columns of the second whisker pair would show no change in linkage, having received the same conditioning stimuli when plasticity was minimal.

\section{Materials and Methods}

Experimental subjects, surgical procedures, and data acquisition. The subjects were five adult male Wistar rats (320-390 gm). Physiological procedures were identical to those reported in previous publications (Rousche et al., 1999; Petersen and Diamond, 2000; Erchova et al., 2002). Surgical anesthesia was induced by urethane $(1.5 \mathrm{gm} / \mathrm{kg}$ body weight, i.p.) and maintained at a constant level by monitoring hindpaw withdrawal, corneal reflex, and respiratory rate and administering extra doses $(10 \%$ of original dose) as necessary. The $10 \times 10$ grid consisted of 1.5 mm-long electrodes with a $400 \mu \mathrm{m}$ interelectrode distance. The array was implanted at a depth of $1200-1300 \mu \mathrm{m}$, corresponding to an infragranular layer recording site, presumably layer $\mathrm{V}$. This depth was selected because it contains a rich plexus of horizontal fibers interconnecting neighboring barrel columns (Kim and Ebner, 1999).

Array placement was examined in histological sections. At termination of the recording session, subjects were perfused with saline and $4 \%$ paraformaldehyde. After postfixation in $20 \%$ sucrose, the cortex was removed, blocked in the frontal plane, and frozen. The block of tissue was cut in $40 \mu \mathrm{m}$ sections in the coronal plane and stained with cresyl violet to verify that the recording sites were in the infragranular layers.

The data acquisition system (Bionic Technologies, Salt Lake City, UT) consisted of a 100-channel amplifier (gain, 5000; signal filtered at bandpass, $250-7500 \mathrm{~Hz}$ ) and digital signal processor (DSP). Voltage thresholds for each channel were set from the computer. The DSP detected when the signal on any channel crossed threshold, extracted $1.5 \mathrm{msec}$ of analog signal ( $0.5 \mathrm{msec}$ before the threshold crossing, $1.0 \mathrm{msec}$ after), and digitized it at 30,000 samples/sec per channel. These waveforms were transmitted to the computer for storage. In off-line analysis, the waveforms emitted by multiunit neuronal clusters were selected for additional analysis (Rousche et al., 1999).

Cross-correlation histograms (CCHs; see below) constructed from spontaneous activity showed that there were no more coincident spikes recorded at neighboring electrodes than would be expected considering the ongoing bursty firing patterns. This rules out the possibility of "cross talk" between the neurons in clusters recorded at adjacent electrodes: the activity of the neuronal cluster recorded at one electrode was never recorded at nearby electrodes (Mirabella et al., 2001; Erchova et al., 2002).

Whisker stimulation and response maps. The 100-microelectrode array permitted us to form "response maps" (Petersen and Diamond, 2000), which are an estimate of the functional representation across the entire array of a single whisker. Before the conditioning protocol (see below), individual whiskers were stimulated $3 \mathrm{~mm}$ from their base using a piezoelectric wafer (Morgan Matroc, Bedford, $\mathrm{OH}$ ) driven by square-wave voltage pulses (A.M.P.I., Jerusalem, Israel). The mapping stimulus, a 100 $\mu \mathrm{m}$ upward deflection released after $100 \mathrm{msec}$, was delivered 50 times, once per second, for each whisker of interest $\left(\alpha, \beta, \gamma, \delta, \mathrm{A}_{1-4}, \mathrm{~B}_{1-4}, \mathrm{C}_{1-6}\right.$, $\left.\mathrm{D}_{1-6}, \mathrm{E}_{1-6}\right)$. The response magnitude at each channel was quantified as the average number of spikes during the $100 \mathrm{msec}$ interval after the upward whisker deflection minus the average number of spikes during the $100 \mathrm{msec}$ interval preceding the deflection. The Wilcoxon sum rank statistical test was used to determine the significance of the response at each channel to a given whisker by comparing the firing rate in the interval $100 \mathrm{msec}$ after stimulus onset to the spontaneous firing rate with the $95 \%$ confidence level; the receptive field of the neuronal cluster at each channel was the set of whiskers that produced a significant response. The principal whisker was identified as the whisker that evoked the largest response. The same data were used to determine the cortical representation of a single whisker; this was the union of all the channels at which the whisker of interest evoked a significant response. Response maps were generated by displaying response values according to the arrangement of electrodes (Petersen and Diamond, 2000).

Additional response maps were acquired for the paired stimulation of "conditioned" whiskers. Each whisker pair received 120 stimuli presented every 2 sec. Stimuli alternated between whisker pairs and total acquisition time was $\sim 5 \mathrm{~min}$. These maps were collected once before conditioning and then $10-15$ and $25-30$ min after conditioning. Peristimulus time histograms (PSTHs) with $1 \mathrm{msec}$ bins were calculated for each neuronal cluster in the time interval from $20 \mathrm{msec}$ before the stimulus onset to $100 \mathrm{msec}$ after. To make statistical comparisons, PSTHs were then averaged across subjects. Average PSTHs of burst-conditioned clusters and of interburst-conditioned clusters were compared for four time periods: $0-10,10-20,20-50$, and $50-100 \mathrm{msec}$ after stimulus onset using the Wilcoxon sum rank test.

Conditioning procedure. We used a 50 min sensory conditioning protocol in which one electrode in the 100-electrode grid was used as a monitor of cortical state: each spike at this electrode was used as a trigger for two pairs of whisker stimuli. Spikes at the trigger channel were linked to the whisker stimulators only during the conditioning period. The time interval between the triggering spike and the stimulus differed for the two whisker pairs: a stimulus generator inserted a $1 \mathrm{msec}$ delay for one pair ("burst-conditioned" whisker pair), whereas it inserted a 300 msec delay for the other pair ("interburst-conditioned" whisker pair). Because bursts usually last $\sim 100 \mathrm{msec}$ and occur at $\sim 1-2 \mathrm{~Hz}$, the delay of 300 msec maximized the probability that the second stimulus occurred during interburst intervals. This delay also minimized the likelihood that EPSPs or IPSPs induced by stimulation of the first whisker pair could directly influence the response to the second whisker pair: inhibition and subthreshold excitation after a whisker deflection peak $\sim 20 \mathrm{msec}$ after stimulus onset and last $\sim 100-120 \mathrm{msec}$, as judging by the time course of two-whisker interactions (Simons, 1985; Shimegi et al., 1999, 2000; 


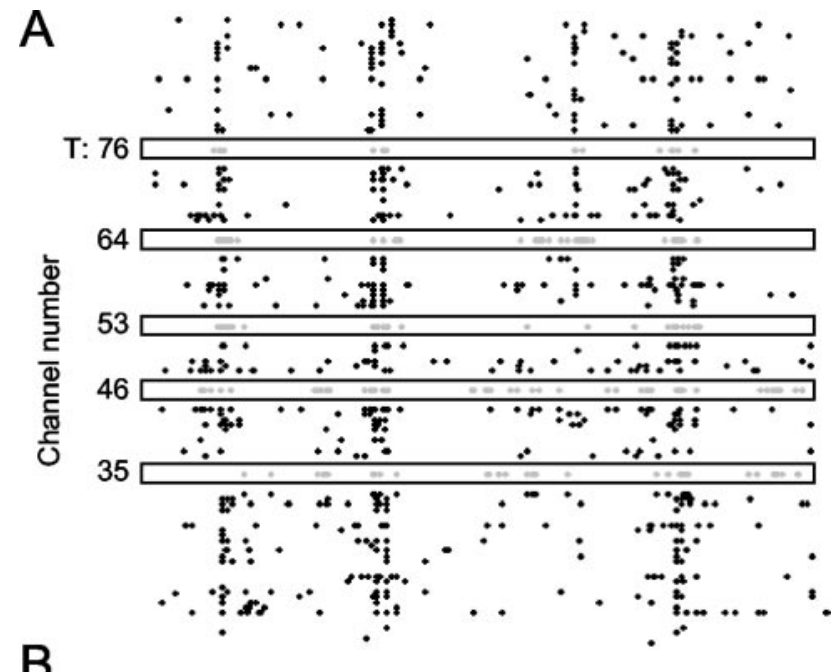

B
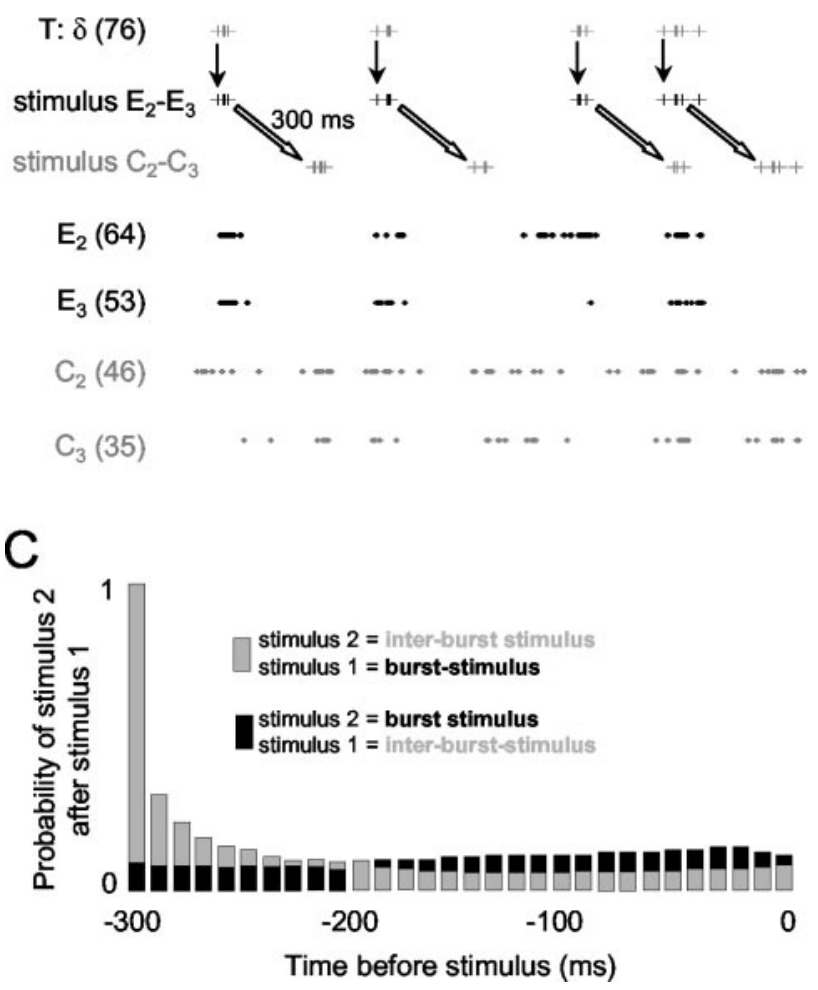

Figure 2. Cortical firing patterns and the conditioning procedure. A, Activity for a 2 sec period at electrodes 1-100 is shown during the conditioning procedure for experiment R20. Spikes at the trigger channel (n.76), at electrodes 53 and 64 (columns $\mathrm{E}_{3}$ and $\mathrm{E}_{2}$, respectively) and at electrodes 35 and 46 (columns $C_{3}$ and $C_{2}$, respectively), are shown as gray dots in black boxes. $B$, Conditioning stimuli are shown for the same 2 sec period of activity. In the top trace, spike times at electrode 76 are shown as vertical gray bars. Immediately below, times of the $E_{2}-E_{3}$ stimulus are shown as vertical bars. Each event at electrode 76 triggered an $E_{2}-E_{3}$ whisker stimulus with a 1 msec delay. Further below times of the $\mathrm{C}_{2}-\mathrm{C}_{3}$ stimulus are shown; note the identical temporal structure as the $\mathrm{E}_{2}-\mathrm{E}_{3}$ stimulus, but with a $300 \mathrm{msec}$ delay. The bottom four traces show spike trains recorded in the burst-conditioned cortical columns $\left(E_{2}-E_{3} ;\right.$ black $)$ and the interburst-conditioned cortical columns $\left(\mathrm{C}_{2}-\mathrm{C}_{3} ;\right.$ gray $)$. Spike trains in the burst-conditioned cortical columns were temporally correlated with the stimuli, whereas spike trains in the interburst-conditioned cortical columns were more dispersed in time. C, Relative timing between stimuliat the burst-conditioned and interburst-conditioned whisker pairs summated across all five experiments. Gray histogram bars show the probability that an interburst stimulus will follow a burst stimulus with a given delay, whereas the black histogram bars show the probability that a burst stimulus will follow an interburst stimulus with a given delay. The histogram value of 1 is determined by the conditioning protocol whereby interburst stimuli always followed burst-triggered stimuli after a 300 msec delay. The other values of the histogram are an outcome of the complex firing patterns at the trigger electrode. Note that at delays of $<\sim 100 \mathrm{msec}$, when stimulus interactions might be pronounced, the probabilities were approximately equivalent.
Erchova et al., 2002) as well as intracellular and calcium imaging data (Moore and Nelson, 1998; Higley and Contreras, 2003; Derdikman et al., 2003).

To completely rule out the possibility of direct suppressive effects of the burst-triggered stimulus, we performed additional checks. To generate response maps before conditioning, each whisker pair was stimulated 120 times with $1 \mathrm{sec}$ between deflections, and from this stimulation paradigm, we could measure cortical responses to a given whisker pair during bursts and interburst intervals without interaction with the other whisker pair. Burst onsets and offsets were detected objectively using the algorithm of Erchova et al. (2002). Averaged across the set of five experiments, the ratio of cortical response magnitude to stimuli presented during bursts versus those presented during interburst intervals was 1.27, confirming previous findings that cortical sensory responses are enhanced during bursts (Erchova et al., 2002). The ratio of cortical responses to burst versus interburst stimuli during conditioning was nearly identical (1.29). This observation argues against the possibility that there were strong lateral interactions in the cortex between successive stimuli and suggests that fluctuations in cortical state are sufficient to explain the lower response to paired whisker stimuli during interburst intervals.

A second relevant point is that whereas interburst stimuli followed burst-triggered stimuli by $300 \mathrm{msec}$, very commonly burst-triggered stimuli followed interburst stimuli by a similar interval. In Figure $2 B$, note that many of the $\mathrm{E}_{2}-\mathrm{E}_{3}$ (burst) stimuli were delivered within a few hundred milliseconds after the $\mathrm{C}_{2}-\mathrm{C}_{3}$ (interburst) stimuli. Figure $2 \mathrm{C}$ shows the relative timing of the burst stimuli and interburst stimuli accumulated across all five experiments. Here, it is evident that at intervals of $<\sim 100-120 \mathrm{msec}$, when one would be expect a maximum inhibitory effect of the preceding stimulus, there was no greater probability of the burst stimulus preceding the interburst stimulus.

The spike triggered whisker-pairing procedure is illustrated in Figure 2 for a $2 \mathrm{sec}$ conditioning period. In this case (experiment R20), electrode 76 was selected as the trigger channel $(T) ; E_{2}-E_{3}$ and $C_{2}-C_{3}$ were the whisker pairs selected for the conditioning protocol. During conditioning, stimulation of whisker pair $\mathrm{E}_{2}-\mathrm{E}_{3}$ was triggered with a 1 msec delay after each spike at electrode 76 , whereas stimulation of whisker pair $\mathrm{C}_{2}-\mathrm{C}_{3}$ was triggered with a $300 \mathrm{msec}$ delay after each spike at electrode 76 . The conditioning stimulus was a square wave of $100 \mu \mathrm{m}$ amplitude in the upward direction and a duration of $10 \mathrm{msec}$. A raster plot of spikes acquired at all 100 electrodes is shown in Figure $2 \mathrm{~A}$. At the trigger electrode, spike times are indicated by gray dots, as are the spike times at channels 64 and 53 (in columns $\mathrm{E}_{2}$ and $\mathrm{E}_{3}$ ) and at channels 46 and 35 (in columns $\mathrm{C}_{2}$ and $\mathrm{C}_{3}$ ). Below (Fig. $2 \mathrm{~B}$ ), spike times at the trigger electrode (T) are shown as gray vertical bars. Vertical downward arrows point to spike-triggered stimulus times (black vertical bars) for whiskers $E_{2}-E_{3}$. Diagonal white arrows point to the stimulus times for whiskers $\mathrm{C}_{2}-\mathrm{C}_{3}$ (gray vertical bars), which occurred after a $300 \mathrm{msec}$ delay. Note that the intrinsic temporal patterns for the two sets of stimuli were identical but phase shifted. Spikes recorded at the electrodes 64 and 53 (located in columns $\mathrm{E}_{2}$ and $\mathrm{E}_{3}$ ) and at electrodes 46 and 35 (located in columns $\mathrm{C}_{2}$ and $\mathrm{C}_{3}$ ) are also shown in black and gray, respectively.

Criteria for inclusion of data. Data from a given experiment were accepted if two sets of criteria were met, the first related to the cortical representations of the whiskers of interest and the second related to the spontaneous activity at the trigger channel.

(1) The five whiskers of interest (the two pairs of whiskers stimulated during the conditioning period, as well as the principal whisker of the neurons at the trigger electrode) had cortical representations similar in magnitude and spatial extent. Moreover, the representation of one whisker pair was segregated from the representation of the other pair, guaranteeing that the conditioning stimuli delivered to a given whisker pair did not produce spikes in the neurons associated with the other whisker pair. These conditions were verified during the conditioning period and by the use of mapping stimuli, as shown here for experiment R20 (Fig. 3).

Finally, none of the whiskers that received the conditioning stimuli were part of the surround receptive field of the neurons at the trigger channel, according to the criteria given in the preceding section. This 
ensured that, during the conditioning protocol, the sensory stimuli did not evoke spikes at the trigger channel, which would in turn produce a whisker stimulus, in a self-sustaining cycle.

(2) It was necessary that the trigger channel act as a reliable gauge of the cortical network state (i.e., the spikes at the trigger channel were temporally aligned with the spikes throughout barrel cortex). This is illustrated for experiment R20 in Figure 4. A $4 \mathrm{sec}$ period of preconditioning spontaneous activity at electrodes $1-100$ is shown. Spikes recorded at the trigger channel (electrode n.76) are given by gray points (Fig. $4 A$ ). It is evident that activity at the selected channel provided a good signal of the overall cortical bursting patterns: periods of increased discharge at the trigger channel occurred at the exact times when the entire network was more active. When spikes were summated across all channels to form a histogram with $50 \mathrm{msec}$ bins (Fig. 4B), trigger channel spikes were distributed close to histogram peaks. To quantify the bursting pattern during the course of the conditioning procedure, we used an algorithm to automatically identify the occurrence of bursts according to the temporal clustering of spikes (Erchova et al., 2002). Bursts identified by this algorithm are shown by horizontal dashes and shading beneath the histogram (Fig. $4 B$ ). Note that the neurons at channel n.76 fired at least one spike during every population burst.

Because it was not possible to completely verify both sets of requirements on-line during the collection of data, many experiments were excluded only after conclusion of the data analysis. Stimulus sites for the five accepted experiments are given in Table 1.

Timing of conditioning stimuli in relation to cortical bursts. The conditioning procedure depends on the temporal characteristics of the cortical bursting pattern. To quantify the bursting pattern during the course of the conditioning procedure, a burst detection algorithm (Erchova et al., 2002) was applied to the collective neuronal activity recorded at all electrodes, excluding the four electrodes in the cortical columns of the stimulated whiskers and all channels immediately surrounding these (this ensured that burst statistics were not influenced by the stimuli). A summary is given in Table 2 . On average, bursts lasted $\sim 150 \mathrm{msec}$. Thus, the delay interval of $300 \mathrm{msec}$ led to a high probability that the second stimulus would be delivered after the conclusion of the burst; hence the term "interburst" stimulus. In fact, whereas $55-70 \%$ of the 1 msec delay sensory stimuli were delivered during bursts, just $7-44 \%$ of the $300 \mathrm{msec}$ delay sensory stimuli were delivered during bursts. Comparing the last two entries in Table 2, we note that, on average, the number of stimuli delivered during bursts was more than five times greater for the burstconditioned whisker pair than for the interburst-conditioned whisker pair.

Because the total number of stimuli applied to the two whisker pairs across the entire conditioning period was equal, and because the intrinsic temporal patterns of the stimulus trains (e.g., the sequence of interstimulus intervals) were equal, any differential cortical plasticity must result from the differential state of cortex at the time of burst-conditioned versus interburst-conditioned stimuli.

Cross-correlation analysis. Background activity was acquired in two 10 min blocks before the conditioning procedure and in two 10 min blocks after the conditioning: $0-10 \mathrm{~min}$ after conditioning and $15-25 \mathrm{~min}$ after conditioning. To characterize the connectivity between neuronal clusters during spontaneous activity, we used cross-correlation analysis (Palm et al., 1988; Eggermont, 1992). For each pair of neuronal clusters, a $\mathrm{CCH}$ with 5 msec bins extending 40 bins before and after the central bin (total, 81 bins) was constructed for a $10 \mathrm{~min}$ period of background activity. To allow comparison across neuron pairs with different firing rates, $\mathrm{CCHs}$ were normalized such that their scaling corresponded to the correlation coefficient (Palm et al., 1988; Aertsen et al., 1989; Eggermont, 1992). The cross-correlation index was defined as the average coefficient value ex-
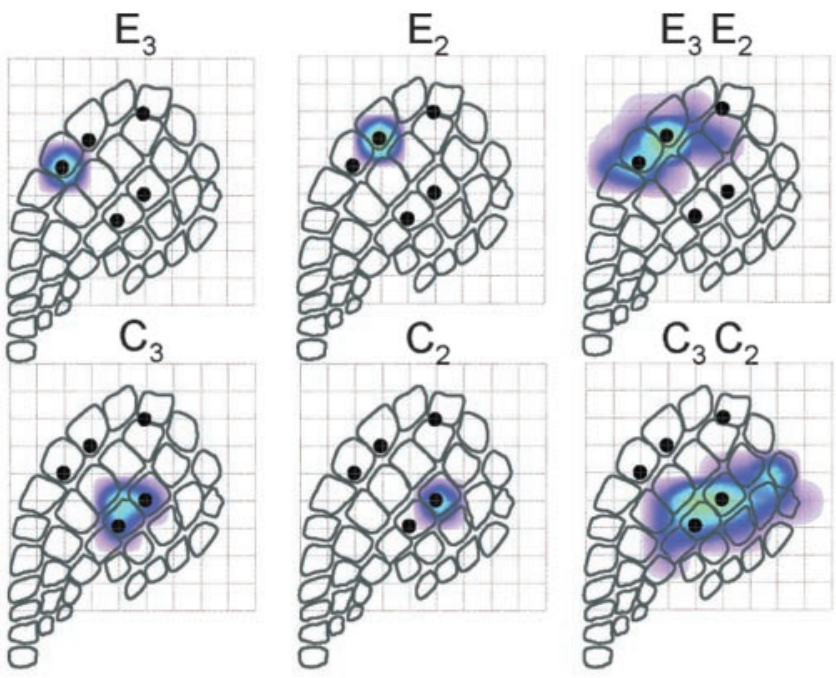

Figure 3. Cortical representation of the five whiskers of interest (experiment R20). In this color scheme, white corresponds to (1)
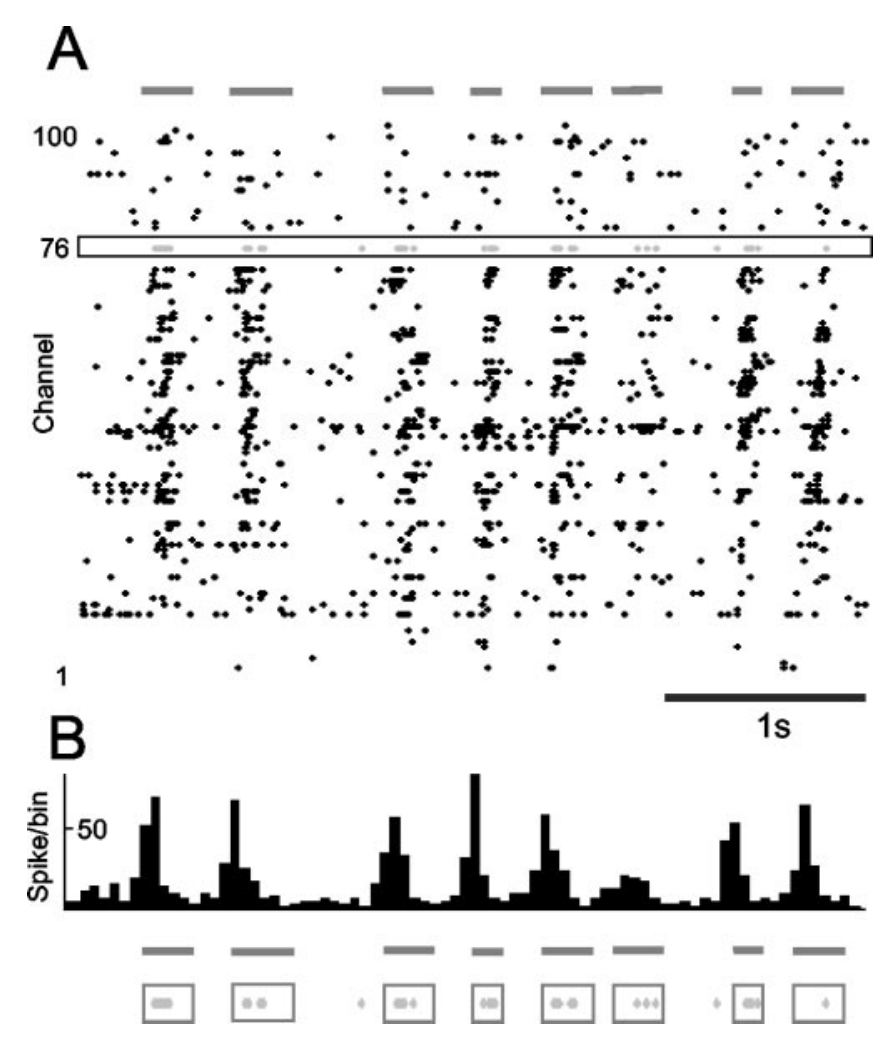

Figure 4. The trigger channel reliably reported cortical network state. A, Spontaneous activity before the conditioning period. Spikes at the trigger channel (gray points at electrode n.76) occur mainly during the bursts of spikes that extend across the entire electrode array. $B$, Histogram formed by spikes summated across all channels. Horizontal lines below the histogram indicate bursts of activity quantitatively defined by whole-array spike density. In the lowest traces, it is clear that nearly every spike at the trigger electrode (gray points) occurred during the whole-array bursts. Every burst included at least one spike from the trigger electrode.

tending from two bins before to two bins after the central bin (total, five bins of $5 \mathrm{msec}$ each). The values during and after conditioning were normalized by the mean value during the $10 \mathrm{~min}$ period of preconditioning background activity.

To determine the connectivity between neuronal clusters present during stimulus-evoked activity, joint PSTHs (JPSTHs) (Aertsen et al., 1989; Gerstein et al., 1989) were constructed on the basis of 120 stimuli deliv- 
Table 1. Trigger and stimulus sites

\begin{tabular}{llll}
\hline $\begin{array}{l}\text { Experiment } \\
\text { number }\end{array}$ & $\begin{array}{l}\text { Principal whisker } \\
\text { at trigger channel }\end{array}$ & $\begin{array}{l}\text { Whiskers stimulated } \\
\text { at 1 msec delay }\end{array}$ & $\begin{array}{l}\text { Whiskers stimulated } \\
\text { at } 300 \text { msec delay }\end{array}$ \\
\hline R15 & $D_{1}$ & $\mathrm{C}_{2}$ and $\mathrm{C}_{3}$ & $\mathrm{E}_{2}$ and $\mathrm{E}_{3}$ \\
R17 & $\mathrm{D}_{1}$ & $\mathrm{C}_{2}$ and $\mathrm{C}_{3}$ & $\mathrm{E}_{2}$ and $\mathrm{E}_{3}$ \\
R18 & $\delta$ & $\mathrm{E}_{2}$ and $\mathrm{E}_{3}$ & $\mathrm{C}_{2}$ and $\mathrm{C}_{3}$ \\
R19 & $\mathrm{D}_{1}$ & $\mathrm{C}_{2}$ and $\mathrm{C}_{3}$ & $\mathrm{E}_{2}$ and $\mathrm{E}_{3}$ \\
R20 & $\delta$ & $\mathrm{E}_{2}$ and $\mathrm{E}_{3}$ & $\mathrm{C}_{2}$ and $\mathrm{C}_{3}$ \\
\hline
\end{tabular}

ered to each pair of whiskers. First, the "raw" JPSTH was constructed by counting the number of joint spikes between the two neuronal clusters in the interval $0-60 \mathrm{msec}$ after stimulus onset ( $30 \times 30$ grid of $2 \mathrm{msec}$ bins). The raw JPSTHs were then corrected by subtracting a "predicted" JPSTH, constructed as the product of the two individual PSTHs. The predicted JPSTH is an estimate of the number of joint spikes that would occur if the two neuronal clusters were independent. The corrected JPSTH, then, is an estimate of the stimulus-induced interaction between the neuronal clusters. The statistical test was based on comparing each bin in the raw JPSTH with the value of the same bin in the predicted JPSTH. Assuming that the coincidence value of the predicted JPSTH is the outcome of a Poisson process, one can calculate the probability of the observed coincidence occurring by chance. This is commonly called the Poisson surprise. The surprise values for each bin were also summed and smoothed to determine the significance of the difference between the raw JPSTH and the predicted JPSTH. JPSTH bins were also summed along the diagonal to create a conventional cross-correlogram. Crosscorrelograms under different conditions were compared using ANOVA on ranks, followed by Dunn's paired comparison.

\section{Results}

\section{Gating of stimulus-induced plasticity}

The chief finding is that the degree of plasticity induced by sensory inputs was determined by the cortical network state at the precise time of stimulus delivery. Activity at one electrode in the 100-electrode grid acted as a monitor of cortical state: each spike at this electrode was used as a trigger for two pairs of whisker stimuli. One whisker pair was stimulated $1 \mathrm{msec}$ after each trigger spike, so the paired sensory inputs were integrated when the cortical network was excitable (burst-conditioned whisker pair). The second whisker pair was stimulated $300 \mathrm{msec}$ after each trigger spike, so the paired sensory inputs were integrated when the cortical network was likely to be less excitable (interburstconditioned whisker pair). Over the course of the 50 min period, burst conditioning caused an increase in the functional connectivity between the barrel columns receiving paired input. In contrast, interburst conditioning caused no significant modification in the functional connectivity between the cortical barrel columns receiving paired input. Because the total number of stimuli delivered to the two pairs of whiskers as well as the temporal patterning of the stimuli was equal, the different degree of intracortical modification must be attributed to rapid modulations in the modifiability of cortex. We will present these findings by illustrating one experiment (R20) and then showing that its main conclusions were valid across experiments.

\section{Cortical modifications induced by burst conditioning}

For experiment R20, Figure $5 A$ shows the cross-correlation index between neurons in barrel columns $\mathrm{E}_{2}$ and $\mathrm{E}_{3}$ (black points), between neurons in barrel columns $\mathrm{C}_{2}$ and $\mathrm{C}_{3}$ (gray points), and between nonstimulated control barrel columns $\mathrm{B}_{2}$ and $\mathrm{B}_{3}$ (white points). The index was measured over 1 min blocks. During the preconditioning period $(0-10 \mathrm{~min})$, the strength of cross-correlation between the $\mathrm{E}_{2}-\mathrm{E}_{3}$ and $\mathrm{C}_{2}-\mathrm{C}_{3}$ barrel columns was equal. At $t=$ $10 \mathrm{~min}$, the conditioning period began, during which neurons in barrel columns $\mathrm{E}_{2}$ and $\mathrm{E}_{3}$ underwent burst conditioning, whereas neurons in barrel columns $\mathrm{C}_{2}$ and $\mathrm{C}_{3}$ underwent interburst conditioning (Fig. $2 B$ ). For both stimulated pairs, but not for the nonstimulated control pair, the amount of correlated activity increased immediately, as would be expected because of the paired whisker stimulation. At $t=30 \mathrm{~min}$, the correlation index for the $\mathrm{E}_{2}-\mathrm{E}_{3}$ pair rose dramatically, whereas the correlation index for the $C_{2}-C_{3}$ pair remained at a constant level. By the conclusion of the $50 \mathrm{~min}$ conditioning paradigm $(t=60 \mathrm{~min})$, the stimulus-induced correlation was approximately twice as great for the burst-conditioned neurons (barrel columns $\mathrm{E}_{2}-\mathrm{E}_{3}$ ) as for the interburst-conditioned neurons (barrel columns $\mathrm{C}_{2}-\mathrm{C}_{3}$ ).

Conditioning terminated at $t=60 \mathrm{~min}$. The cross-correlation index for the interburst-conditioned neurons immediately returned to the preconditioning level, but the cross-correlation index for the burst-conditioned neurons remained elevated. The modification in effective connectivity for the burst-conditioned neurons was still present at $t=85 \mathrm{~min}, 25 \mathrm{~min}$ after the conclusion of the conditioning protocol.

Changes in intracortical connectivity for this experiment are summarized in Figure $5 B$. CCHs were constructed separately from five 10 min intervals, labeled a-e in Figure 5A. Before conditioning (interval a) and shortly after the onset of conditioning (interval b), the CCHs for the burst-conditioned neurons, the interburstconditioned neurons, were equivalent. By the final $10 \mathrm{~min}$ period of the conditioning period (interval c), the $\mathrm{CCH}$ for the burstconditioned neurons had grown larger, whereas that for the interburst-conditioned neurons was practically unchanged. The heightened synchrony between spikes of the burst-conditioned neurons remained after the conclusion of conditioning (intervals $\mathrm{d}$ and e), whereas the synchrony between the interburst-conditioned neurons returned to the preconditioning level. The strength of correlation between the nonstimulated barrel columns $\left(\mathrm{B}_{2}-\mathrm{B}_{3}\right)$ was not significantly affected.

The difference in correlation strength between the $\mathrm{E}_{2}-\mathrm{E}_{3}$ pair and the $\mathrm{C}_{2}-\mathrm{C}_{3}$ pair were quantified by statistical analyses (ANOVA on ranks test, followed by Dunn's paired comparison). The values of the cross-correlation index during $10 \mathrm{~min}$ of preconditioning spontaneous activity were not significantly different for the two pairs of electrodes $(Z=0.03 ; p=0.49)$. After the conditioning procedure, the same statistical test revealed a significant difference for both $10 \mathrm{~min}$ intervals $(Z=2.05 ; Z=1.93 ; p<$

Table 2. Physiological characterization of the conditioning procedure

\begin{tabular}{lllll}
\hline & R20 & R15 & R17 & R18 \\
\hline Burst rate (Hz) & 1.10 & 1.40 & 0.75 & 0.97 \\
Mean burst duration [SD] (msec) & $120[44]$ & $245[71]$ & $187[88]$ & $126[44]$ \\
Percentage of spikes on trigger channel within bursts & 58.5 & 72.7 & 70.7 & 63.0 \\
Number of stimuli per whisker pair generated in 50 min & 21,778 & 28,971 & 27,045 & 12,113 \\
Percentage of stimuli presented within burst for 1 msec delay pair & 54.6 & 70.4 & 65.4 & 73.7 \\
Percentage of stimuli presented within burst for 300 msec delay pair & 10.8 & 43.6 & 19.1 & 25,322 \\
\hline
\end{tabular}


0.05). Cross-correlation values for the neurons at a given pair of electrodes were then compared before and after conditioning. For the burst-conditioned neurons, values of the cross-correlation indices $0-10$ and $15-25 \mathrm{~min}$ after conditioning $(t=60-70$ and 75-85 min) were significantly greater $\left(F_{(3,1)}=15.03 ; p<0.05\right)$ than those measured before conditioning. For the interburst-conditioned neurons, values of the cross-correlation index did not differ significantly $\left(F_{(3,1)}=0.083 ; p>\right.$ $0.4)$ from those measured before conditioning during either of the postconditioning intervals.

Sensory responses also were modified by the conditioning protocol. Paired whisker stimuli were applied before conditioning and then at 70-75 and $85-90 \mathrm{~min}$. For the same experiment as described above, R20, Figure $6 A$ shows the PSTHs evoked by stimulation of the burst-conditioned (top) and interburst-conditioned (bottom) whisker pairs. Each PSTH combines the activity from both electrodes of a pair. Before conditioning (left), the PSTHs appeared similar; when subdivided into four intervals $(0-10 \mathrm{msec}$, the interval during which thalamocortical inputs are predominant; and 10-20, 20-50, and 50-100 $\mathrm{msec}$ ), the values in each interval for burstconditioned versus interburst-conditioned PSTHs were not different $(0-10 \mathrm{msec}, t=$ $0.11 ; 10-20 \mathrm{msec}, t=0.15 ; 20-50 \mathrm{msec}, t=$ $0.05 ; 50-100 \mathrm{msec}, t=0.10 ; p>0.4 ; n=$ $3440)$. Compared with preconditioning values, after conditioning (middle and right), the PSTHs recorded at the interburstconditioned cortical columns were unchanged, whereas the PSTHs recorded at the burst-conditioned columns revealed increased sensory responses in late poststimulus intervals. More precisely, response magnitude was unchanged for the $0-10$ msec interval but increased for time intervals $10-20,20-50$, and $50-100 \mathrm{msec}(0-10$ msec: $t=0.67, p=0.2 ; 10-20$ msec: $t=$ $1.86, p<0.02 ; 20-50$ msec: $t=2.52, p<$ $0.01 ; 50-100$ msec: $t=5.1, p<0.01 ; n=$ 3440). From the same data set, Figure $6 B$ shows response maps to paired whisker stimuli. Using the outline of the preconditioning maps as reference, there appears to have been an expansion in the territory activated by the burst-conditioned whiskers (top) and less so for the interburstconditioned whiskers (bottom).

Cross-correlation results averaged across all five rats are given in Figure 7. To allow comparison between cases, the crosscorrelation index for each rat was normalized to a value of 1.0 for the preconditioning period. Throughout the course of conditioning, the average value of the cross-correlation index was greater for the burst-conditioned electrodes (black points) than for the interburst-conditioned electrodes (gray points). For the interburst-conditioned electrodes, there was no trend for a pro-
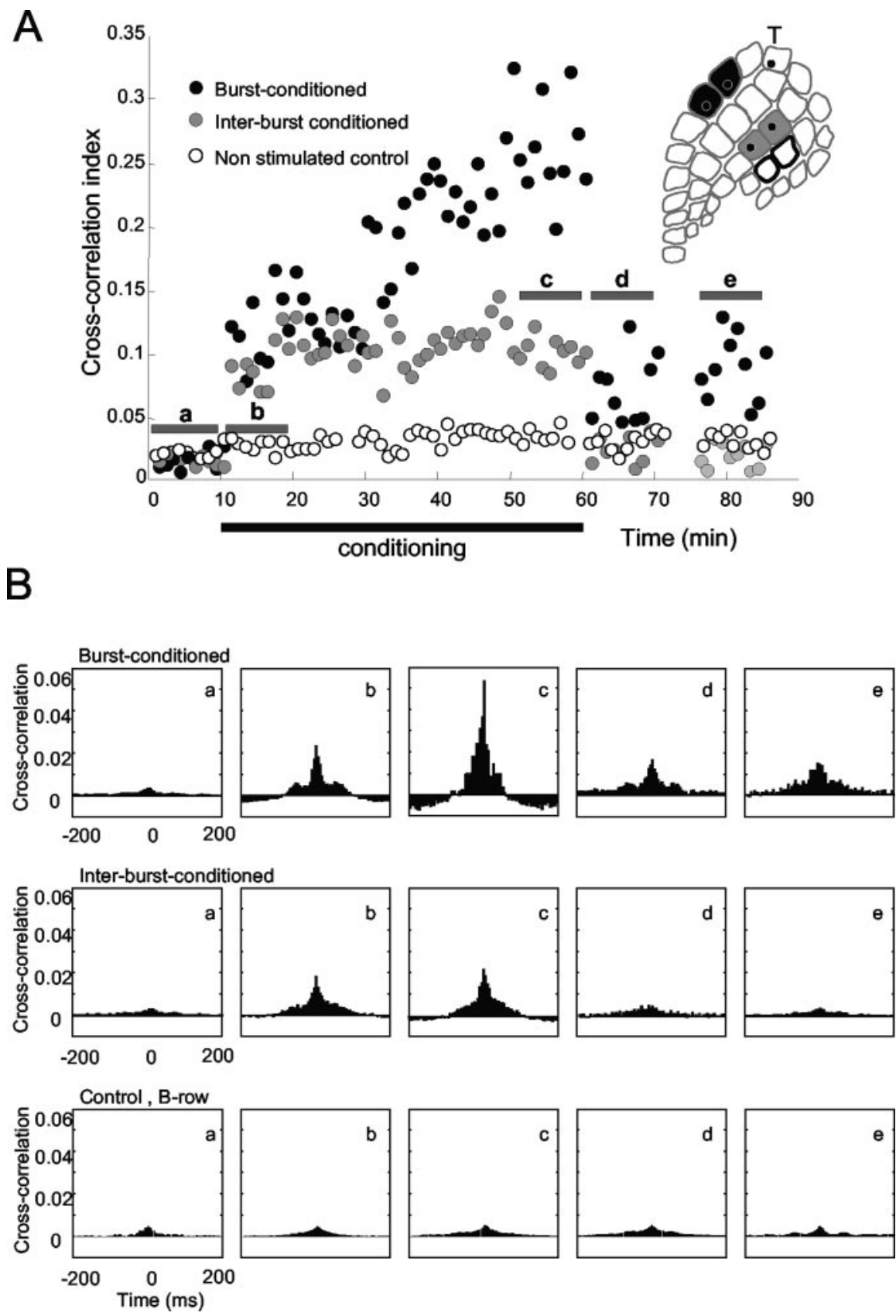

Figure 5. State-dependent changes in intercolumnar connectivity. $A$, Cross-correlation index between neurons in burstconditioned columns ( $E_{2}$ and $E_{3}$; black points), between neurons in inter-burst-conditioned columns $\left(C_{2}\right.$ and $C_{3} ;$ gray points), and between neurons in nonstimulated control columns ( $B_{2}$ and $B_{3}$; white points). $B$, CCHs constructed from activity measured during intervals a-e in $A$. CCHs for the columns receiving burst-conditioning pairing increased during conditioning and remained elevated. The site of the trigger electrode is indicated by $T$.

gressive increase in correlation beyond that present at the onset of conditioning. In contrast, $20 \mathrm{~min}$ after the onset of the conditioning, the average correlation strength between the burstconditioned electrodes began to rise (as was true for the individual case shown in Fig. 5A) and continued to increase steadily.

At $60 \mathrm{~min}$, the conditioning terminated, and the average crosscorrelation during spontaneous activity remained about twice as great for the burst-conditioned neurons as for the interburstconditioned neurons. The latter returned nearly to the preconditioning level. The pairing-induced modification in effective connectivity was still present at $t=85 \mathrm{~min}, 25 \mathrm{~min}$ after the conclusion of the conditioning protocol. 


\section{A Pre-conditioning}
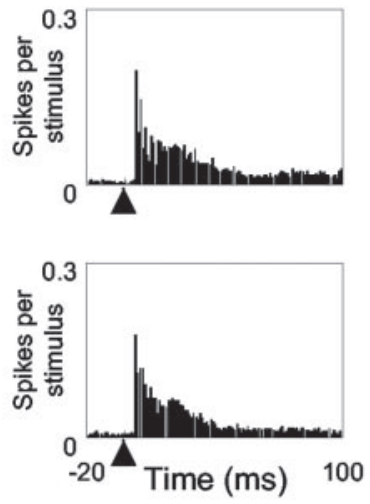

\section{B Pre-conditioning}
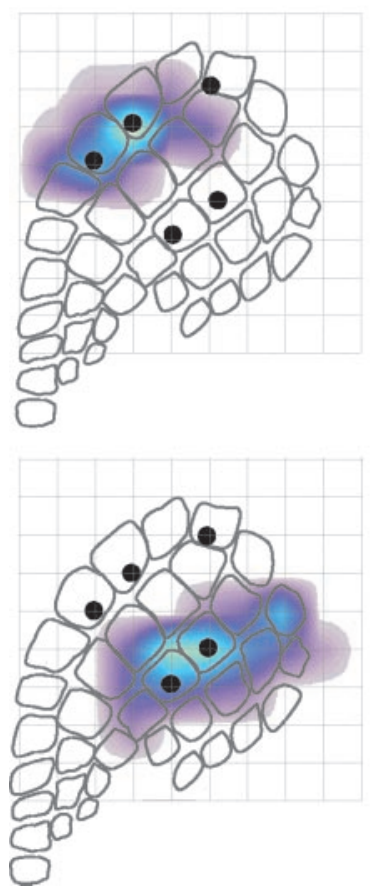

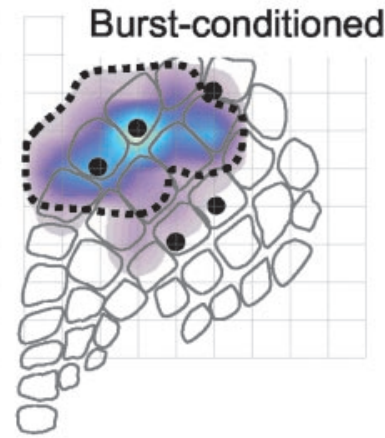

10-15 min post Burst-conditioned

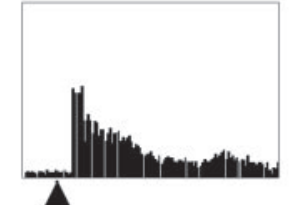

Inter-burst-conditioned

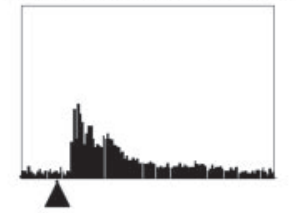

10-15 min post Burst-conditioned
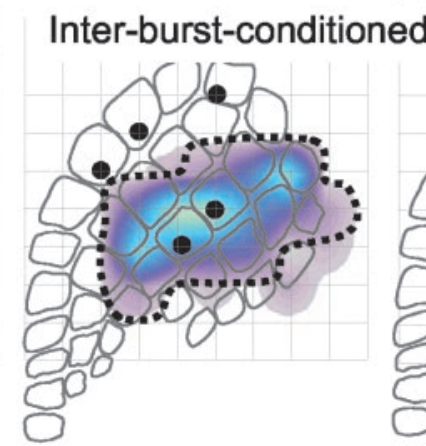

25-30 min post
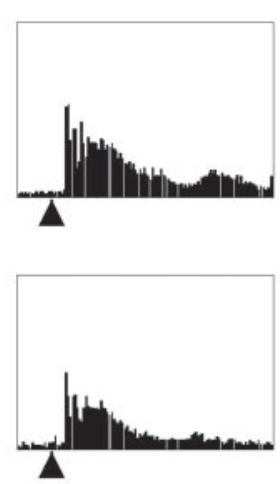

25-30 min post

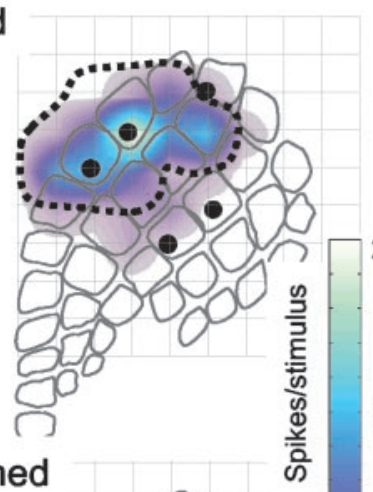

2.0 1.0

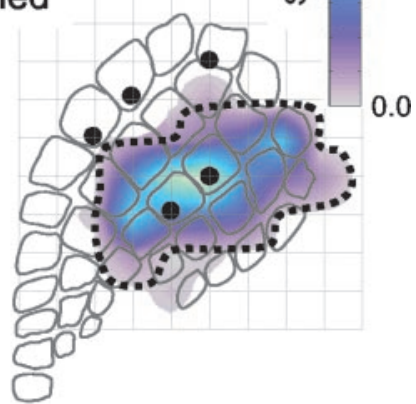

Figure 6. Changes in sensory responses attributable to conditioning in experiment R20. A, PSTHs from both electrodes in the burst-conditioned pair (top) and interburst-conditioned pair (bottom) generated by stimulation of the corresponding whisker pair. The arrowhead indicates stimulus onset time. Conditioning did not change the PSTH profile for the interburst-conditioned neurons, but for the burst-conditioned neurons, a larger response was evident, particularly after $\sim 10 \mathrm{msec}$. The bin size is $1 \mathrm{msec}$. $B$, Cortical territories activated by the burst-conditioned whisker pair (top) and the interburst-conditioned whisker pair (bottom) before conditioning (left) and after conditioning (middle and right). The dotted line indicates the territory activated before conditioning. For the burst-conditioned whisker pair, there appears to be an expansion of the activated cortical territory.

comparison was made using raw (nonnormalized) cross-correlation values, and differences between neurons at the two pairs of electrodes were not found for any of the subjects $(Z=0.023$; $p=0.998)$. Conditioning induced statistically significant differences in the normalized correlation index in both intervals (0-10 and $15-25 \mathrm{~min})$ after the conclusion of conditioning $(Z=2.51$, $p<0.01 ; Z=1.71, p=0.04)$.

Specificity of the cortical modification Two simple control analyses prove that the changes were spatially specific and temporally specific. First, to show that the modifications in functional connectivity were limited to the neurons of the two burstconditioned columns, in each experiment we calculated the average crosscorrelation index for the neuron cluster at one burst-conditioned electrode against the neuron cluster at one interburstconditioned electrode. For example, for case R20, the "anti-paired" neurons were in barrel columns $\mathrm{C}_{2}-\mathrm{E}_{2}$ and $\mathrm{C}_{3}-\mathrm{E}_{3}$. The cross-correlation value for anti-paired columns remained at a constant level before, during, and after conditioning (Fig. 8A), indicating that the modifications in functional connectivity were specific to the two sets of neurons paired during cortical bursts. Moreover, the change in connectivity occurred only if neuronal co-activity was caused by sensory inputs: neurons at the trigger electrode and the burstconditioned electrodes were coactive (because sensory inputs to the burstconditioned columns were evoked by spikes at the trigger electrode), but connectivity between them did not change (data not shown).

Second, to show that the increased cross-correlation reflected precise cofiring of the burst-conditioned neurons rather than simply increased overall firing (which can also elevate the crosscorrelation index), we calculated the average firing rate for the neuronal clusters at both pairs of electrodes (Fig. $8 \mathrm{~B}$ ). During

Statistical tests performed on the averaged data set (ANOVA on ranks test, followed by Dunn's paired comparison) substantiated the observed changes in neuronal cross-correlation. For neurons at the burst-conditioned electrodes, values of the crosscorrelation indices $0-10$ and $15-25 \mathrm{~min}$ after conditioning were significantly greater $\left(F_{(3,5)}=5.01 ; p<0.05\right)$ than those measured before conditioning. For the interburst-conditioned neurons, values of the cross-correlation indices were not significantly greater $\left(F_{(3,5)}=0.03 ; p>0.3\right)$ than the preconditioning value for either of the $10 \mathrm{~min}$ post-conditioning intervals.

We also made direct comparisons between burst-conditioned and interburst-conditioned cross-correlation index values. During $10 \mathrm{~min}$ of spontaneous activity preceding conditioning, the conditioning, the firing rate was higher for the burst-conditioned neurons than for the interburst-conditioned neurons, presumably because the sensory stimulus combined with the ongoing bursts to create a greater number of spikes (Erchova et al., 2002). The crucial point, however, is that at the conclusion of the conditioning period, the firing rates for the burst-conditioned and the interburst-conditioned neurons were indistinguishable from each other $(p>0.9)$. Thus, the higher cross-correlation index observed for the burst-conditioned neurons was not attributable to a greater number of spikes, but rather was as a result of a more precise synchrony of the same number of spikes. In summary, burst conditioning did not change the firing rate but changed the firing pattern. 


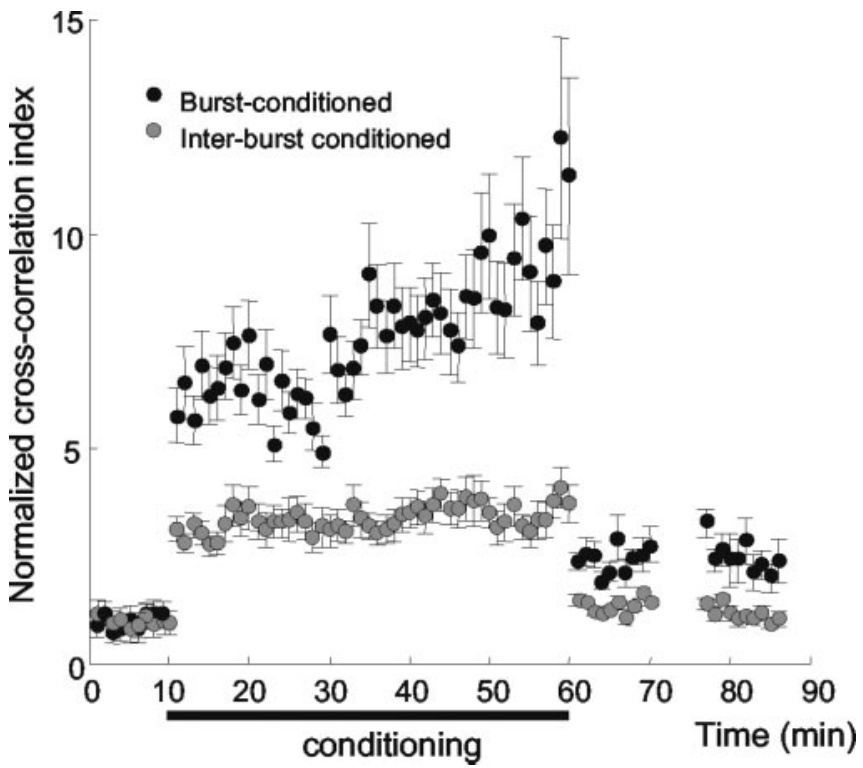

Figure 7. Changes in intercolumnar connectivity for all five rats. On average, the normalized cross-correlation index for the columns receiving burst-conditioning pairing (black) increased during conditioning and remained elevated. Error bars show SE of measurements.

Changes in the correlated responses between paired columns To determine whether the conditioning procedure caused changes in the stimulus-induced cross-correlation between cortical columns, we constructed JPSTHs between neurons in burstconditioned and interburst-conditioned columns. JPSTH values were also summated along the diagonal to form correlograms and reveal the strength of connectivity across the entire response period. Any JPSTH value $>0$ is referred to as "positive" because it indicates that the observed number of joint spikes for that bin was larger than that predicted if the stimulus-induced responses of the two columns were independent (i.e., it exceeded the value given by multiplying the separate PSTHs). Before conditioning, there was, on average, a positive stimulus-induced correlation between the neurons in adjacent cortical columns, both for the burst-conditioned and the interburst-conditioned neurons (Fig. $9 A$ ). As expected, there was no significant difference between the levels of co-activation for the two pairs of cortical columns before conditioning ( $Z=0.05 ; p=0.02$ ). For both pairs, stimulusinduced correlations began $\sim 6-8 \mathrm{msec}$ after stimulus onset, suggesting that the earliest synchrony was attributable to common thalamic input. Positive correlations continued throughout the measured response period; these late joint spikes could derive from common thalamic input or from intercolumnar relay. The positive interactions occurred mostly with time differences of $<10-15 \mathrm{msec}$ (i.e., within $\sim 10-15$ msec of the diagonal of the JPSTHs).

The conditioning procedure led to changes in stimulusinduced connectivity (Fig. 9B). The overall cross-correlation for neuronal clusters in the burst-conditioned columns was greater than that for neuronal clusters in the interburst-conditioned columns $10-15$ and 25-30 min after conditioning ended $\left(F_{(3,2)}=\right.$ 15.3 and $F_{(3,2)}=13.2, p<0.05$, respectively). The JPSTH differences appear to result from a combination of heightened coincident activity in the burst-conditioned columns, together with decreased coincident activity in the interburst-conditioned columns. Considered together with PSTH data from Figure 6A, the results indicate that changes in the functional connectivity between cortical columns were manifest not only in their sponta-
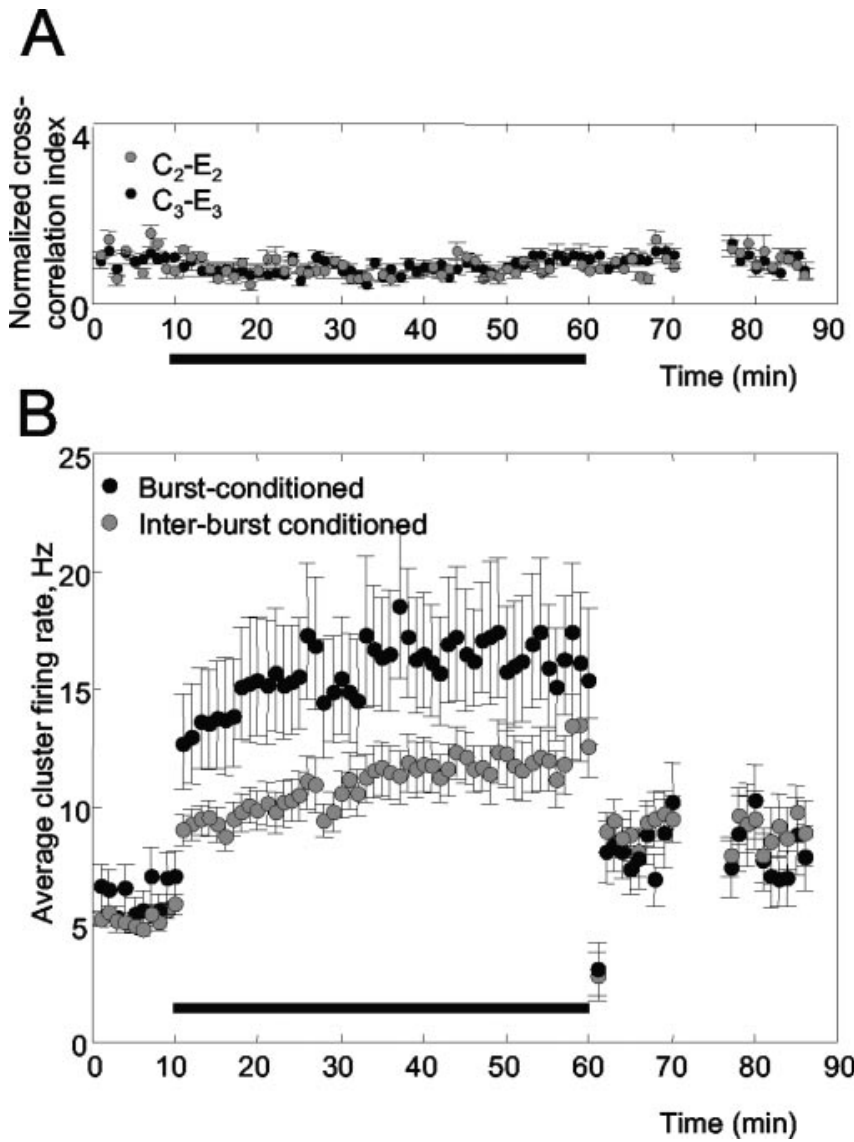

Figure 8. Analyses to control for the specificity of connectivity changes (experiment R20). $A$, During conditioning, cross-correlation increased only between the burst-conditioned cortical columns $\left(E_{2}-E_{3}\right)$ and not between $E_{2}$ or $E_{3}$ and either of the interburst-conditioned columns $\left(C_{2}-\right.$ $E_{2}$ and $C_{3}-E_{3}$ are shown). $B$, Firing rates for burst-conditioned and interburst-conditioned neurons increased during conditioning but then returned nearly to the baseline preconditioning level. Increased cross-correlation for burst-conditioned columns was thus attributable to temporally precise spikes rather than simply a greater number of spikes.

neous activity but also in their sensory-evoked activity. More precisely, the conditioning procedure produced a disparity in intercolumnar connectivity, favoring the columns receiving paired inputs during cortical bursts relative to the columns receiving the same quantity of paired inputs during interburst intervals.

\section{Discussion}

The results presented here show that the cortex in anesthetized animals can be modified by sensory input patterns only during discrete, intermittent intervals. Stimuli delivered during bursts, short intervals of elevated spontaneous activity, were effective in modifying cortical connectivity, whereas stimuli delivered in the intervals between bursts, although matching in number and temporal pattern, were ineffective. In all five experiments, the conditioning paradigm produced a comparable degree of plasticity in the burst-triggered channels, despite the widely varying number of stimuli per whisker pair generated in 50 min (Table 2).

Below, we consider the mechanisms through which the rapid variations in cortical excitability modulate intracortical plasticity and what the functional implications might be.

\section{Plasticity of intracortical connectivity}

In awake attentive animals, training on sensory discrimination tasks (Jenkins et al., 1990; Recanzone et al., 1992) or periods of 
A Pre-conditioning Burst-conditioned
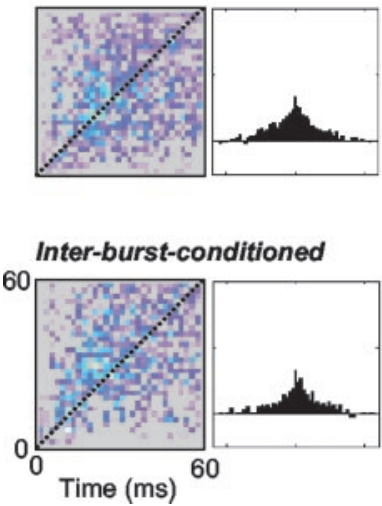

B $10-15$ min post conditioning

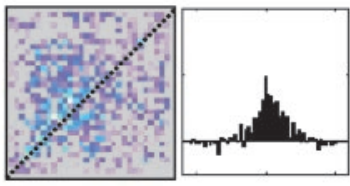

coincidences/trial

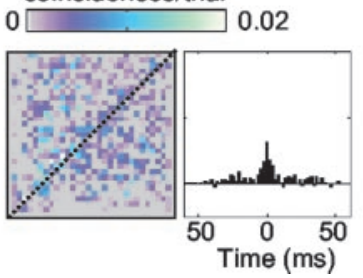

C $25-30$ min post conditioning
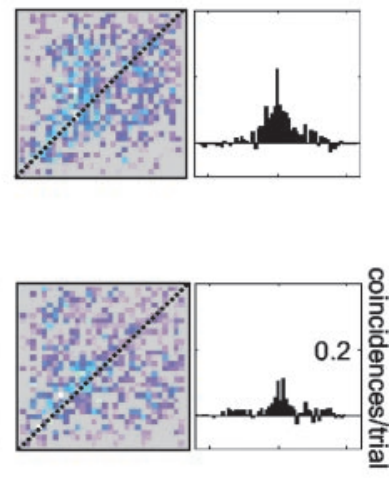

Figure 9. Changes in stimulus-induced connectivity. The colored plots show JPSTHs after normalization by subtraction of the predicted JPSTH from the raw JPSTH. The cross-correlogram to the right of each JPSTH represents the sum of coincidences along the diagonal axis. $A-C$ show data collected at successive stages of the experiment from burst conditioning (top) and interburst conditioning (bottom).

altered sensory experience (Diamond et al., 1993, 1994; Lebedev et al., 2000) cause changes in somatosensory cortical receptive fields and cortical maps. Modification of effective intracortical connectivity has been proposed as one of the candidate mechanisms to account for the plasticity of sensory cortex (ArmstrongJames et al., 1994; Wang et al., 1995). The clearest evidence for the hypothesis comes from an experiment in which, in alert monkeys, a high level of contingency between the firing of two neurons in the auditory cortex (spontaneous activity of one neuron triggered an immediate sensory input to the second neuron) altered the connectivity between the pair of neurons, as measured by cross-correlation (Ahissar et al., 1992).

A deeper understanding of the mechanisms of receptive field plasticity in alert, behaving animals has been hindered by (1) the lack of precise stimulus control and (2) the difficulty in identifying and recording from the appropriate sets of neurons. Although these can be overcome by studying anesthetized animals, an even more important limit is then introduced: the cortex of anesthetized animals seems to be non-plastic unless some external manipulation is included during the stimulation protocol (e.g., basal forebrain stimulation) (Tremblay et al., 1990; Howard and Simons, 1994; Kilgard and Merzenich, 1998), or direct administration of ACh (Lamour et al., 1988; Juliano et al., 1990). Similarly, in cortical in vitro preparations, long-term potentiation in the sensory-motor cortex (a synaptic model for receptive field plasticity) can be induced only by experimentation on immature brains, in which NGF and ACh concentrations are very high (Kimura et al., 1989), through manipulation using exogenous factors such as bicuculline (Artola and Singer, 1987; Hess and Donoghue, 1996) and Mg + concentration (Lee et al., 1991), and through unnatural high-frequency electrical stimulus trains (Dinse et al., 1993).

\section{What is the mechanism for rapid fluctuations in plasticity?}

The main result of the present experiment is that the barrel cortex is modifiable during short intervals that are correlated with a specific phase of the cortical network fluctuation. Our preferred explanation is that neuromodulatory substances, in particular $\mathrm{ACh}$, are released in the sensory cortex during the peak of the cortical burst/pause rhythm and that sensory inputs arriving during that phase can induce modifications in cortical circuitry.
The major source of cholinergic input to neocortex is the nucleus basalis (NB) of the basal forebrain. Sillito and Kemp (1983) suggested that the tonic discharge of NB neurons can cause a sustained increase in ACh release in the cortex and consequent neuronal changes associated with arousal. On the time scale of seconds to minutes, the release of ACh in the cortex is tightly linked to the state of the EEG (Celesia and Jasper, 1966; Szerb, 1967): the release of ACh in the cortex increases markedly during cortical EEG desynchronization (DeFeudis, 1975). Systemic injections of cholinergic agonists produce low-voltage desynchronized EEG, whereas injections of cholinergic antagonists produce high-voltage synchronized EEG (for review, see Longo, 1966). Sensory stimulation causes some basal forebrain neurons to shift from an irregular tonic discharge to a rhythmic burst discharge simultaneously with the change in EEG to a rhythmic discharge (Manns et al., 2000).

Our experiments, however, concern cortical state variations on a much narrower time scale. Although, to date, there is no direct evidence for phasic ACh release timed to peaks of burst periods, basal forebrain/sensory cortex co-firing is present even on this sub-second time scale: individual EEG peaks and troughs (which we assume are the large-scale expression of neuronal cluster bursts) are associated with the firing of basal forebrain neurons (Detari et al., 1999; Duque et al., 2000; for review, see Zaborsky and Duque, 2003). There are two types of basal forebrain neurons, differing in their firing pattern (Nunez, 1996). Tonic neurons display spontaneous single spike firing unrelated to the slow waves. In contrast, bursting neurons $(\sim 60 \%$ of the total) exhibit periodic bursts at $0.3-2 \mathrm{~Hz}$ (matching the typical cortical burst frequency in our data set) (Table 2) that are phaselocked with the EEG slow waves (Nunez, 1996; Manns et al., 2000). At a fine time scale, it appears that basal forebrain activity slightly precedes each cortical EEG peak (Duque et al., 2000).

If the bursting of basal forebrain neurons causes a transient elevation in cortical ACh concentration, this could complement the increased synaptic reliability present during cortical bursts (Lisman, 1997). The picture that is suggested, then, is that under urethane anesthesia cortical bursts are linked to basal forebrain firing and the transient release of ACh combines with the ongoing cortical burst to enable plasticity. Another candidate as a modulatory input, the activity of which is linked to barrel cortex bursts, is the intralaminar thalamic nuclei (Fox and Armstrong-James, 1986).

\section{Locus of modification}

The analyses showed heightened correlated activity between burst-conditioned columns both during spontaneous activity, revealed by simple cross-correlograms, and during whisker stimulation, revealed by JPSTHs. The augmented stimulus-related correlations could reflect either strengthened intercolumnar connectivity or else a more synchronized level of common ascending input to the two columns; we cannot specify the locus of this effect. In other sensory conditioning paradigms, however, cortex is more plastic than its inputs. For example, in primates, synchronous multi-digit stimulation causes increased correla- 
tion between the neurons with paired inputs in the somatosensory cortex but not in the corresponding nucleus of the thalamus (Wang et al., 1995). From the circuitry of the rat somatosensory system, stimulus-induced common input seems unlikely to account for the increased cross-correlation in spontaneous activity after the conditioning period. Changes in common input synchrony specific to the paired cortical columns during spontaneous activity could occur only if the two thalamic barreloids projecting to two cortical columns were active in a more synchronous manner, implying some communication between the barreloids; however, neurons in barreloids have no direct interconnection (Jones, 1985). It is simpler to suggest that repetitive costimulation of two whiskers, timed to coincide with transient increases in cortical plasticity, caused the burst- conditioned cortical barrel columns to become more strongly connected, presumably through Hebbian mechanisms (Hebb, 1949).

Our data set came from infragranular layer recordings. In pilot experiments, we did not detect changes in intercolumnar connectivity when recordings were made from layer IV. This, too, is consistent with the proposal that plasticity is expressed primarily in lateral intracortical connections: layer IV neurons are only weakly connected to neighboring columns, whereas the neurons we sampled here are part of a dense network of lateral connections (Kim and Ebner, 1999). Our experiments add one more piece of evidence (for review, see Lebedev et al., 2000) to support the idea that horizontal connections above and below layer IV are particularly modifiable.

\section{Cortical fluctuations and plasticity}

What the present study may add to the understanding of cortical plasticity is that, even in the anesthetized brain, sensory cortex can be modifiable so long as the sensory input pattern is timed to occur coincident with peaks in intrinsic fluctuations in excitability. The possibility that these findings can be of general relevance is supported by the fact that cortical fluctuations occur in every brain state; their detailed nature depends on the behavioral conditions. We suggest that, in every state, the timing of the peaks and troughs of these variations may modulate the ability of ascending inputs to modify the cortex. In rats, intermittent fluctuations occur in relation to natural behaviors such as whisking and sniffing. We speculate that sensory information is collected and stored in the cortex at discrete time intervals dictated by such fluctuations. Additional confirmation will require the study of unanesthetized animals.

\section{References}

Aertsen AM, Gerstein GL, Habib MK, Palm G (1989) Dynamics of neuronal firing correlation: modulation of "effective connectivity." J Neurophysiol 61:900-917.

Ahissar E, Vaadia E, Ahissar M, Bergman H, Arieli A, Abeles M (1992) Dependence of cortical plasticity on correlated activity of single neurons and on behavioral context. Science 257:1412-1415.

Armstrong-James M, Diamond ME, Ebner FF (1994) An innocuous bias in whisker use in adult rats modifies receptive fields of barrel cortex neurons. J Neurosci 14:6978-6991.

Artola A, Singer W (1987) Long-term potentiation and NMDA receptors in rat visual cortex. Nature 330:649-652.

Celesia GG, Jasper HH (1966) Acetylcholine released from cerebral cortex in relation to state of activation. Neurology 16:1053-1063.

DeFeudis FV (1975) Amino acids as central neurotransmitters. Annu Rev Pharmacol 15:105-130.

Derdikman D, Hildesheim R, Ahissar E, Arieli A, Grinvald A (2003) Imaging spatiotemporal dynamics of surround inhibition in the barrels somatosensory cortex. J Neurosci 23:3100-3105.

Detari L, Rasmusson DD, Semba K (1999) The role of basal forebrain neu- rons in tonic and phasic activation of the cerebral cortex. Prog Neurobiol 58:249-277.

Diamond ME, Armstrong-James M, Ebner FF (1993) Experiencedependent plasticity in adult rat barrel cortex. Proc Natl Acad Sci USA 90:2082-2086.

Diamond ME, Huang W, Ebner FF (1994) Laminar comparison of somatosensory cortical plasticity. Science 265:1885-1888.

Dinse HR, Recanzone GH, Merzenich MM (1993) Alterations in correlated activity parallel ICMS-induced representational plasticity. NeuroReport 5:173-176.

Duque A, Balatoni B, Detari L, Zaborszky L (2000) EEG correlation of the discharge properties of identified neurons in the basal forebrain. J Neurophysiol 84:1627-1635.

Eggermont JJ (1992) Neural interaction in cat primary auditory cortex. Dependence on recording depth, electrode separation, and age. J Neurophysiol 68:1216-1228.

Erchova IA, Lebedev MA, Diamond ME (2002) Somatosensory cortical neuronal population activity across states of anaesthesia. Eur J Neurosci 15:744-752.

Fox K, Armstrong-James M (1986) The role of the anterior intralaminar nuclei and $\mathrm{N}$-methyl D-aspartate receptors in the generation of spontaneous bursts in rat neocortical neurones. Exp Brain Res 63:505-518.

Freiwald WA, Kreiter AK, Singer W (1995) Stimulus dependent intercolumnar synchronization of single unit responses in cat area 17. NeuroReport 6:2348-2352.

Gerstein GL, Bedenbaugh P, Aertsen MH (1989) Neuronal assemblies. IEEE Trans Biomed Eng 36:4-14.

Hebb DA (1949) The organisation of behavior: a neuropsychological theory. New York: Wiley.

Hess G, Donoghue JP (1996) Long-term potentiation and long-term depression of horizontal connections in rat motor cortex. Acta Neurobiol Exp (Warsz) 56:397-405.

Higley MJ, Contreras D (2003) Nonlinear integration of sensory responses in the rat barrel cortex: an intracellular study in vivo. J Neurosci 23:10190-10200.

Hoffman KL, McNaughton BL (2002) Sleep on it: cortical reorganization after-the-fact. Trends Neurosci 25:1-2.

Howard 3rd MA, Simons DJ (1994) Physiologic effects of nucleus basalis magnocellularis stimulation on rat barrel cortex neurons. Exp Brain Res 102:21-33.

Jenkins WM, Merzenich MM, Ochs MT, Allard T, Guic-Robles E (1990) Functional reorganization of primary somatosensory cortex in adult owl monkeys after behaviorally controlled tactile stimulation. J Neurophysiol 63:82-104.

Jones EG (1985) The thalamus. New York: Plenum.

Juliano SL, Ma W, Bear MF, Eslin D (1990) Cholinergic manipulation alters stimulus-evoked metabolic activity in cat somatosensory cortex. J Comp Neurol 297:106-120.

Kilgard MP, Merzenich MM (1998) Cortical map reorganization enabled by nucleus basalis activity. Science 279:1714-1718.

Kim U, Ebner FF (1999) Barrels and septa: separate circuits in rat barrels field cortex. J Comp Neurol 408:489-505.

Kimura F, Nishigori A, Shirokawa T, Tsumoto T (1989) Long-term potentiation and N-methyl-D-aspartate receptors in the visual cortex of young rats. J Physiol (Lond) 414:125-144.

Kreiter AK, Singer W (1996) Stimulus-dependent synchronization of neuronal responses in the visual cortex of the awake macaque monkey. J Neurosci 16:2381-2396.

Lamour Y, Dutar P, Jobert A, Dykes RW (1988) An iontophoretic study of single somatosensory neurons in rat granular cortex serving the limbs: a laminar analysis of glutamate and acetylcholine effects on receptive-field properties. J Neurophysiol 60:725-750.

Lebedev MA, Mirabella G, Erchova I, Diamond ME (2000) Experiencedependent plasticity of rat barrel cortex: redistribution of activity across barrel-columns. Cereb Cortex 10:23-31.

Lee SM, Weisskopf MG, Ebner FF (1991) Horizontal long-term potentiation of responses in rat somatosensory cortex. Brain Res 544:303-310.

Lisman J (1997) Bursts as a unit of neural information: making unreliable synapses reliable. Trends Neurosci 20:38-43.

Llinas RR, Pare D (1991) Of dreaming and wakefulness. Neuroscience 44:521-535. 
Llinas R, Ribary U, Contreras D, Pedroarena C (1998) The neuronal basis for consciousness. Philos Trans R Soc Lond B Biol Sci 353:1841-1849.

Longo VG (1966) Behavioral and electroencephalographic effects of atropine and related compounds. Pharmacol Rev 18:965-996.

Manns ID, Alonso A, Jones BE (2000) Discharge properties of juxtacellularly labeled and immunohistochemically identified cholinergic basal forebrain neurons recorded in association with the electroencephalogram in anesthetized rats. J Neurosci 20:1505-1518.

Mirabella G, Battiston S, Diamond ME (2001) Integration of multiplewhisker inputs in rat somatosensory cortex. Cereb Cortex 11:164-170.

Moore CI, Nelson SB (1998) Spatio-temporal subthreshold receptive fields in the vibrissa representation of rat primary somatosensory cortex. J Neurophysio 1 80:2882-2892.

Nunez A (1996) Unit activity of rat basal forebrain neurons: relationship to cortical activity. Neuroscience 72:757-766.

Palm G, Aertsen AM, Gerstein GL (1988) On the significance of correlations among neuronal spike trains. Biol Cybern 59:1-11.

Pare D, Llinas R (1995) Conscious and pre-conscious processes as seen from the standpoint of sleep-waking cycle neurophysiology. Neuropsychologia 33:1155-1168.

Petersen RS, Diamond ME (2000) Spatial-temporal distribution of whisker-evoked activity in rat somatosensory cortex and the coding of stimulus location. J Neurosci 20:6135-6143.

Recanzone GH, Merzenich MM, Dinse HR (1992) Expansion of the cortical representation of a specific skin field in primary somatosensory cortex by intracortical microstimulation. Cereb Cortex 2:181-196.

Rousche PJ, Petersen RS, Battiston S, Giannotta S, Diamond ME (1999) Examination of the spatial and temporal distribution of sensory cortical activity using a 100-electrode array. J Neurosci Methods 90:57-66.

Shimegi S, Ichikawa T, Akasaki T, Sato H (1999) Temporal characteristics of response integration evoked by multiple whisker stimulations in the barrel cortex of rats. J Neurosci 19:10164-10175.

Shimegi S, Akasaki T, Ichikawa T, Sato H (2000) Physiological and anatom- ical organization of multiwhisker response interactions in the barrel cortex of rats. J Neurosci 20:6241-6248.

Siegel JM, Rogawski MA (1988) A function for REM sleep: regulation of noradrenergic receptor sensitivity. Brain Res 472:213-233.

Sillito AM, Kemp JA (1983) Cholinergic modulation of the functional organization of the cat visual cortex. Brain Res 289:143-155.

Simons DJ (1985) Temporal and spatial integration in the rat SI vibrissa cortex. J Neurophysiol 543:615-635.

Steriade M, Gloor P, Llinas RR, Lopes de Silva FH, Mesulam MM (1990) Report of IFCN Committee on Basic Mechanisms. Basic mechanisms of cerebral rhythmic activities. Electroencephalogr Clin Neurophysiol 76:481-508.

Steriade M, Nunez A, Amzica F (1993) Intracellular analysis of relations between the slow $(<1 \mathrm{~Hz})$ neocortical oscillation and other sleep rhythms of the electroencephalogram. J Neurosci 13:3266-3283.

Steriade M, Timofeev I, Grenier F (2001) Natural waking and sleep states: a view from inside neocortical neurons. J Neurophysiol 85:1969-1985.

Szerb JC (1967) Cortical acetylcholine release and electroencephalographic arousal. J Physiol (Lond) 192:329-343.

Timofeev I, Grenier F, Bazhenov M, Sejnowski TJ, Steriade M (2000) Origin of slow cortical oscillations in deafferented cortical slabs Cereb Cortex 10:1185-1199.

Tobler I (2000) Phylogeny of sleep regulation. In: Principles and practice of sleep medicine (Kryger MH, Roth T, Dement WC, eds), pp 72-78. Philadelphia: Saunders.

Tremblay N, Warren RA, Dykes RW (1990) Electrophysiological studies of acetylcholine and the role of the basal forebrain in the somatosensory cortex of the cat. II. Cortical neurons excited by somatic stimuli. J Neurophysiol 64:1212-1222.

Wang X, Merzenich MM, Sameshima K, Jenkins WM (1995) Remodelling of hand representation in adult cortex determined by timing of tactile stimulation. Nature 378:71-75.

Zaborszky L, Duque A (2003) ) Sleep-wake mechanisms and basal forebrain circuitry. Front Biosci 8:1146-1169. 